\title{
Fiber-Optic Add-Drop Device Based on a Silica Microsphere-Whispering Gallery Mode System
}

\author{
Ming Cai, Guido Hunziker, and Kerry Vahala, Member, IEEE
}

\begin{abstract}
An all-optical passive four-port system including a fused silica microsphere and two tapered fibers is proposed and demonstrated for the application as a channel adding-dropping device. It is shown that channels can be selectively exchanged between two fibers by coupling to a whispering gallery mode resonance in the microsphere. Finesse in excess of 11150 is measured for the loaded whispering gallery modes.
\end{abstract}

Index Terms - Fiber optics, finesse, microsphere, quality factor, signal add-drop, whispering gallery mode.

$\mathbf{T}$ HE INTERACTION of optical waves with spherical dielectric microcavities has been a subject of long-term interest. The corresponding electromagnetic eigen-modes are labeled by three indices $(n, l, m)$, where $n$ is the mode order and gives the number of maxima in the radial intensity distribution of the field inside the cavity; $l$ is the angular order number, and $m=-l, \cdots, 0, \cdots, l$, is the azimuthal number. Among these modes, some of the more interesting are associated with a set of indices that have a low value of $n(\sim 1)$, high value of $l(\gg n)$, and $|m| \cong l$. Referred to as whispering gallery modes (WGM's) [1], they are spatially confined near the equator that is normal to the $z$ axis. Whispering gallery modes in fused-silica microspheres have been excited using an evanescent field provided by a prism [2] or a polished optical fiber coupler [3], [4]. Recently, efficient coupling was also demonstrated using a tapered optical fiber [5]. In this letter, we describe a four-port passive channel add-drop device based on the microsphere-fiber coupling mechanism, and demonstrate its feasibility by constructing such a device using dual fiber tapers. Four-port passive add-drop devices based on resonator coupling of semiconductor waveguides [6] or half-block optical couplers [4] have been recently proposed. The devices described here offer another potential approach to this device.

The four-port device is sketched in Fig. 1. Two nearly identical tapered fibers are positioned to tangentially touch opposite sides of an equator of the microsphere. The microsphere sustains WGM resonances at a series of wavelengths $\left\{\lambda_{l, m}\right\}$. Careful positioning of the two fibers at the upper and lower taper-microsphere contact points enables coupling to these resonant modes. The four coupling ports are labeled as

Manuscript received December 1, 1998; revised March 5, 1999.

M. Cai and K. Vahala are with the Department of Applied Physics, California Institute of Technology, Pasadena, CA 91125 USA.

G. Hunziker was with the Department of Applied Physics, California Institute of Technology, Pasadena, CA 91125 USA. He is now with Lucent Technologies, Holmdel, NJ 07733 USA.

Publisher Item Identifier S 1041-1135(99)04251-2.

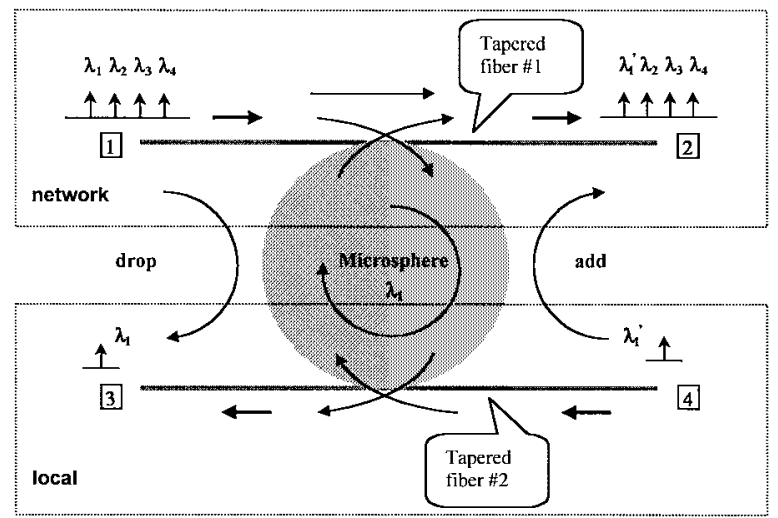

Fig. 1. Schematic of the passive four-port device showing a channel at wavelength $\lambda_{1}$ being dropped from port 3 while another channel at $\lambda_{1}^{\prime}$ is added from port 4 .

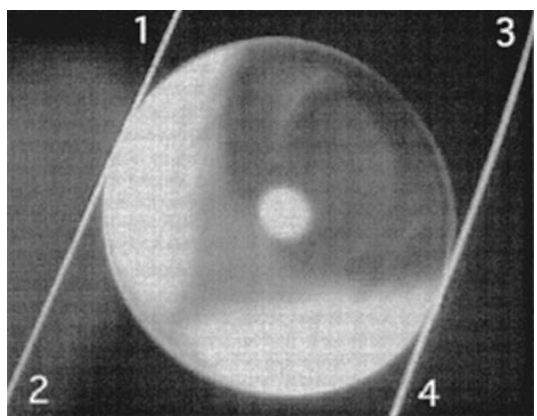

Fig. 2. Photograph of the top view of the microsphere and dual-tapered fiber system. All components shown are fabricated from standard SMF. The microsphere has a diameter of $300 \mu \mathrm{m}$, and the waists of the two fibers are about $3 \mu \mathrm{m}$. All ports have been numbered for reference.

shown. If a signal containing several channels at wavelengths $\lambda_{1}, \lambda_{2}, \lambda_{3}, \lambda_{4}, \cdots$ is input at port 1 , then power transfer to the microsphere and to port 3 will occur for any input channel that is resonant with a WGM ( $\lambda_{1}$ in the figure). Other nonresonant channels travel through the upper fiber past the microsphere with minimal powerloss. Similarly, a channel input at port 4 that is resonant with a WGM would be transferred to the upper fiber, and join the other channels at port 2. Channel adding and dropping therefore can be realized simultaneously.

A magnified photograph of the four-port device is shown in Fig. 2. We used standard optical telecommunication singlemode fiber (SMF) having a refractive index $N$ of 1.43 to fabricate both the microsphere and the tapers. The microsphere had a radius $R$ of $\sim 150 \mu \mathrm{m}$ and was held by a stem (underneath the sphere and not seen in the picture) with 


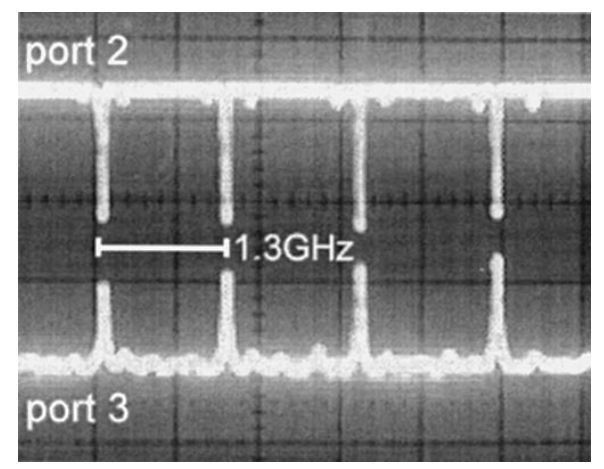

Fig. 3. Power transmission output spectra from port 2 and port 3 (see Fig. 2). The minima and maxima correspond to WGM resonances in the microsphere.

a diameter of $\sim 50 \mu \mathrm{m}$ at the connection point with the sphere. The dual tapers were formed using the same technique described in [5] for fabrication of a single taper. In this case, however, two fibers were placed in parallel and heated simultaneously and uniformly to create tapers having nearly the same size and profile. The waists of the tapers were about $3 \mu \mathrm{m}$.

Two nearly identical photodetectors were used to simultaneously monitor the signals from port 2 and port 3 on an oscilloscope. Typical transmission spectra are shown in Fig. 3. The optical source was a New Focus tunable external-cavity diode laser having a linewidth less than $300 \mathrm{kHz}$. The laser frequency was continuously scanned $(600 \mathrm{~Hz}$ scan frequency) around $1540 \mathrm{~nm}$. The upper trace is the transmission spectrum from port 2, and the lower trace is from port 3 . The frequency spacing between adjacent peaks is about $1.3 \mathrm{GHz}$ in the photograph and corresponds to the frequency splitting of the " $m$ " modal index induced by eccentricity of the sphere.

Port 2 exhibits transmission power minima while port 3 exhibits maxima at resonances. They are displayed using different vertical scales. The power of the maxima in the lower trace was up to $50 \%$ of the input power at Port 1, depending on mode order. Much of this coupling loss is attributed to taper loss (see comment below). The extinction of resonant transmission at port 2 was also measured upon careful adjustment of the position of the tapers and the polarization of the laser. Our best results gave an extinction exceeding $20 \mathrm{~dB}$ when the $Q$ factor was approximately $10^{6}$. The insertion loss for port 1 to port 2 nonresonant mode transmission was tested by monitoring power at port 2 both with and without the sphere contacting the taper. Based on these measurements, a loss of about $3 \mathrm{~dB}$ was measured and determined to be almost completely dominated by the taper loss alone.

Under coupling conditions in which the sphere touched the tapers, the linewidth at half-power $\Delta \nu_{1 / 2}$ of the WGM resonances varied from 20 to $100 \mathrm{MHz}$, corresponding to the quality factors of $1 \times 10^{6}$ to $7 \times 10^{6}$ and a resonator finesse $\left(F=c / 2 \pi N R \Delta \nu_{1 / 2}\right)$ as high as 11150 . Upon removing the second fiber (port 3 and port 4) from the sphere, the linewidth $\Delta \nu_{1 / 2}$ of the transmission minima at port 2 decreased by approximately a factor of 2 , which is quite reasonable considering that the field coupling coefficient and the field attenuation inside the sphere are very small. Higher $Q$ 's of $\sim 10^{7}$ were also observed when the first fiber was pulled away somewhat from the sphere, however, amplitudes of both minima and maxima were then observed to decrease rapidly.

In consideration of the add-drop application, there are several technical challenges concerning the characteristics of the present device. First, both the frequency spacing between adjacent modes and the mode linewidth should be increased substantially. Linewidths in excess of several $\mathrm{GHz}$ would be preferable for telecom applications and mode frequency spacings of several terahertz would be desirable to enable single channel selection in a system carrying in excess of 100 optical channels. The former problem might be solved by using smaller spheres, however, the latter problem will mostlikely require the use highly eccentric resonator structures. In addition, higher extinction values will be required beyond what has been observed. Further studies are now underway that address each of these areas.

In conclusion, we presented an all-fiber four-port passive device in which silica-microsphere WGM resonances provide coupling between two tapered fibers. Results demonstrating the add-drop function were described. Further work is necessary to ascertain whether this device can provide a truly practical approach to the add-drop function, however, the coupling efficiency, extinction, and finesse values in the devices tested so far are encouraging.

\section{REFERENCES}

[1] P. W. Barber and R. K. Chang, Eds., Optical Effects Associated with Small Particles. Singapore: World Scientific, 1988.

[2] M. L. Gorodetsky and V. V. Ilchenko, "High- $Q$ optical whisperinggallery microresonators: Precession approach for spherical mode analysis and emission patterns with prism couplers," Opt. Commun., vol. 113, pp. 133-143, Dec. 1994.

[3] A. Serpenguzel, S. Arnold, and G. Griffel, "Excitation of resonances of microspheres on an optical fiber," Opt. Lett., vol. 20, no. 7, pp. 654-656, 1995.

[4] F. Treussart, N. Dubreuil, J. C. Knight, V. Sandoghdar, J. Hare, V. Lefevre-Seguin, J. M. Raimond, and S. Haroche, "Microlasers based on silica microspheres," Ann. Telecommun., vol. 52, nos. 11-12, pp. 557-568, 1997

[5] J. C. Knight, G. Cheung, F. Jacques, and T. A. Birks, "Phase-matched excitation of whispering gallery mode resonances using a fiver taper," Opt. Lett., vol. 22, no. 15, pp. 1129-1131, 1997.

[6] B. E. Little, J. S. Foresi, G. Steinmeyer, E. R. Thoen, S. T. Chu, H. A. Haus, E. P. Ippen, L. C, Kimerling, and W. Greene, "Ultra-compact $\mathrm{Si}-\mathrm{SiO}_{2}$ microring resonator optical channel dropping filters," IEEE Photon. Technol. Lett., vol. 10, pp. 549-551, Apr. 1998. 